\title{
Efectos de la dependencia al celular en las habilidades sociales de los estudiantes universitarios
}

\author{
Effects of cell phone dependence on the social skills of university students
}

\author{
Diana De la Cruz Sandoval' ${ }^{1}$, Luz Torres Zárate², Elí Yánac Cierto³
}

\begin{abstract}
RESUMEN
Objetivo: Determinar la relación entre dependencia al celular y habilidades sociales de los estudiantes de una universidad privada de Lima Este. Metodología: Se utilizó el diseño no experimental de corte transversal y de tipo correlacional. Asimismo, se empleó un muestreo no probabilístico intencional, incluyendo alumnos de ambos sexos con edades entre 17 a 28 años. Resultados: El $52.1 \%$ de los universitarios se encuentran en riesgo de presentar dependencia al celular, mientras el $22.8 \%$ ya lo presenta frente a un $25.1 \%$ que no. Asimismo, el $56.1 \%$ de los estudiantes evidencian bajo nivel de habilidades sociales, el $29.9 \%$ están en proceso de desarrollarlos y solo el $14 \%$ han desarrollado las habilidades sociales. Además, se encontró relación negativa y altamente significativa entre las variables de estudio (Rho=-,645**; $p<0.01$ ), de igual manera se encontró relación negativa y altamente significativa en las dimensiones de dependencia al celular con el total de habilidades sociales: "abstinencia" (Rho=-,624*; $p<0.01$ ), "abuso y dificultad de controlar el impulso" (Rho=-,526**; $<0.01)$, "problemas ocasionados por el uso excesivo" (Rho=-,595**; $p<0.01)$ y "tolerancia" (Rho=-,569**; p <0.01) Conclusión: La dependencia al celular si se relaciona altamente significativa y de manera inversa con las habilidades sociales. Es decir, a mayor dependencia del celular se asocia un nivel menor de habilidades sociales y viceversa.
\end{abstract}

Palabras claves: Dependencia al celular, Habilidades sociales, universitarios.

\begin{abstract}
Objective: To determine the relationship between cell phone dependency and social skills of the students of a private university in East Lima. Methodology: The non-experimental cross-sectional and correlational type design was used. Likewise, an intentional non-probabilistic sampling was used, including students of both sexes with ages between 17 and 28 years. Results: $52.1 \%$ of university students are at risk of presenting dependence to the cell phone, while $22.8 \%$ already have it, compared to $25.1 \%$ who do not. Likewise, $56.1 \%$ of the students show a low level of social skills, $29.9 \%$ are in the process of developing them and only $14 \%$ have developed social skills. In addition, a negative and significant relationship was found between the study variables (Rho= -, 645, $p<0.01$ ), in the same way a negative and significant relationship was found in the cell dependency dimensions with the total social skills: "abstinence "(Rho= -,624; $p<0.01)$, "abuse and difficulty of controlling the impulse" (Rho= -, 526; $p<0.01$ ), "problems caused by excessive use" (Rho= -, 595; $p<0.01$ ) and "tolerance" (Rho= -, 569; $p$ <.01). Conclusion: Cell dependency is significantly related and inversely related to social skills. That is, a higher level of cell phone dependency is associated with lower level of social skills is and vice versa.
\end{abstract}

Keywords: Cell phone dependence, social skills, university.

\footnotetext{
${ }^{1}$ Centro de Salud Nuevo Bambamarca, Tocache, Perú.

${ }^{2}$ Consultorio privado de psicología, Lima, Perú.

3Universidad Peruana Unión, Lima, Perú.

Orcid ID: 0000-0001-5958-3886
} 


\section{INTRODUCCIÓN}

En la sociedad actual la comunicación virtual ha pasado a convertirse en parte fundamental de la realidad cotidiana (Castells, 2014) produciendo un cambio en la vida de las personas, quienes consideran a este dispositivo como elemento indispensable (Niño, 2013) sobre todo para la Generación Z "nativos digitales" para quienes la tecnología es una forma de estar en el mundo, donde los contactos virtuales son considerados reales a diferencia de los personales (Ozkan y Solmaz, 2015). Frente a esto Ruiz, Sánchez y Trujillo (2015) manifiestan que el uso del teléfono celular cada vez es más variado, lo que incrementan considerablemente las posibilidades de dependencia al dispositivo de una manera importante.

Los estudios a nivel mundial evidencian porcentajes considerables de dependencia al celular, siendo los países más afectados en Europa: España con el 2,8\% hasta el 19,5\% de abuso a estos dispositivos siendo los jóvenes y de sexo femenino quienes presentarían mayores problemas (Carbonell et al., 2012). En Latinoamérica esta problemática se ve reflejada en Colombia, donde el $36 \%$ de los encuestados utilizan el celular entre 1 y 5 horas diarias, seguidos por un 35\% que utiliza entre 6 y 10 horas, y el $18 \%$ entre 11 y 15 horas y finalmente el $11 \%$, presentan uso promedio entre 16 y 20 horas diarias (Arias et al. 2011). A su vez, en Argentina el $80 \%$ de los jóvenes encuestados entre 18 a 25 años, presentaba abuso al celular (Telesur, 2017). Mientras que en el Perú el Instituto Nacional de Salud Ambiental Honorio Delgado-Hideyo Noguchi refiere que del $30 \%$ al $40 \%$ de las personas que utilizan teléfonos móviles sufren de adicción, siendo una de las señales de adicción el "síndrome de abstinencia" (Agencia Peruana de Noticias [Andina], 2013). Así mismo, en Arequipa un estudio con población universitaria, determinó que el $8,1 \%$ de mujeres y el 3,4\% de varones presentaba síntomas de adicción al celular (Morales, 2012).

Esta problemática es considerada como adicción sin drogas, en las que se presentan conductas repetitivas con el objetivo de minimizar la tensión perjudicando a la persona por el incremento de la intensidad, frecuencia y cantidad de dinero invertido afectando las relaciones a nivel familiar, social y laboral (Echeburúa y Corral, 1994).
La población más susceptible son los jóvenes quienes emplean continuamente el celular que se ha convertido en un dispositivo imprescindible en sus vidas por los múltiples beneficios que les proporciona (Gaspar, 2016).

Frente a esta realidad, es necesario identificar el efecto que tiene la dependencia al celular sobre las habilidades sociales como lo menciona Viracocha (2017) la dependencia al celular ha traído consecuencias a nivel social en el que se evidencia déficit en el desarrollo de las habilidades sociales en un contexto de relación interpersonal como ser más fácil expresar los sentimientos a través de redes sociales que cara a cara o prefiriendo usar el teléfono celular que relacionarse con los seres queridos. En esta misma línea, Tejeiro, Pelegrina y Gómez (2009) refieren que las consecuencias presentadas en el uso excesivo de este dispositivo llevan a alejarse de las amistades, reduciendo las interacciones, haciéndose menos sociable y poniendo en riesgo las habilidades sociales.

Para Gismero las habilidades sociales son "EI conjunto de respuestas verbales y no verbales, y situacionalmente específicas, a través de las cuales un individuo expresa en un contexto interpersonal sus necesidades, sentimientos, preferencias, opiniones o derechos sin ansiedad excesiva y de manera asertiva, respetando todo ello en los demás, que trae como consecuencia el auto reforzamiento y maximiza la probabilidad de conseguir refuerzo externo" (citado por Garcés, Feliciano y Santana, 2018).

Salvador (2017) en un estudio realizado en Lima encontró que existe relación inversa entre adicción a internet y habilidades sociales (Rho $=-0.313$ ) en estudiantes de secundaria. Concluyendo que los estudiantes con adicción presente tienen mayor prevalencia en los estudiantes cuya categoría de habilidades sociales son promedio bajo (29.5\%). Es decir, a mayor prevalencia de adicción a internet menor desarrollo de habilidades sociales. Por su parte, Mejía, Paz y Quinteros (2014) en su investigación de adicción a Facebook y habilidades sociales encontraron que existe una correlación inversa altamente significativa $(r=-0.256 ; p<0.01)$ lo que demostraría que un uso excesivo de Facebook disminuye las habilidades sociales o viceversa. Así mismo, Chambi y Sucari (2017) en su estudio entre dependencia al celular y habilidades sociales encontraron correlación, aunque en un nivel bajo $(r=-.2 .05, p<0.05)$. 
Estas investigaciones sugieren que los estudiantes que presentan adicción a las redes sociales, las que pueden ser frecuentadas desde el celular, tienden a presentar dificultad en desarrollar las habilidades sociales, las cuales son susceptibles de aprender y desarrollarse (Bisquerra 2003).

Por ello, el objetivo de esta investigación es determinar si existe relación significativa entre dependencia al celular y habilidades sociales en estudiantes de una universidad privada de Lima este.

\section{METODOLOGÍA}

En esta investigación se hizo uso del diseño no experimental, porque no se manipularon las variables, de corte transversal porque se recolectó la información en un tiempo establecido y de alcance correlacional, ya que busca analizar la relación entre las dos variables (Hernández, Fernández \& Baptista, 2010).

\section{Participantes}

Participaron 351 estudiantes de una universidad privada de Lima Este, del primero a quinto año de la Escuela de Psicología, seleccionados a través de un muestreo no probabilístico intencional. Se incluyó a estudiantes de ambos sexos con edades entre 17 a 28 años, quienes completaron los test de forma voluntaria.

\section{Instrumento}

\section{Test de dependencia al móvil (TDM)}

Fue creado por Chóliz y Villanueva en el año 2010. Está constituido por cuatro dimensiones: abstinencia, control de impulso, problema por uso excesivo y tolerancia. Los ítems son 22 en total los que están divididos en dos partes: el primero con 10 ítems que se responden mediante "Nunca" hasta "Siempre" y el segundo de 12 ítems y se responde "totalmente en desacuerdo" hasta "totalmente de acuerdo" ambos mediante una escala de tipo Likert que oscila entre 0 a 4 puntos. Respecto a su fiabilidad esta resultó con un valor alpha de Cronbach de 0,94 (Chóliz \& Villanueva, 2010). Fue estandarizado en Arequipa por Gamero et al. (2015) obteniendo una fiabilidad y validez del instrumento elevada, con el alfa de Cronbach, $\alpha=0,929$.

\section{Escala de habilidades sociales (EHS)}

Fue creado por Gismero en el año 2005. Está constituido por 7 dimensiones: autoexpresión de situaciones sociales, defensa de los propios derechos como consumidor, expresión de enfado o disconformidad, decir no y cortar interacciones, hacer peticiones e iniciar interacciones positivas con el sexo opuesto. Contiene 33 ítems con una escala de respuesta de tipo Likert que oscila entre 1 a 4 puntos. De los cuales, 28 ítems están redactadosm acerca de la falta de aserción o bajo en habilidades sociales, y 5 son aspectos positivos. En el Perú fue adaptado por Cesar Ruiz Alva en el año 2006. Demostrando una fiabilidad de alfa de Cronbach de 0.88 considerado como alta (Mejía et al., 2014).

\section{RESULTADOS}

La muestra estuvo conformada por 351 participantes, siendo el $73.8 \%$ de sexo femenino y el $26.2 \%$ de sexo masculino. Los estudiantes entre 20 a 24 años conforman el 58.1\%, mientras el grupo entre 16 a 19 años conforman el $30.8 \%$ y finalmente el grupo de 25 a más años conforman el $11.1 \%$. Respecto a los lugares de procedencia se tiene estudiantes de la costa en un $58.1 \%$, de la sierra $30.8 \%$, de la selva $8 \%$ y del extranjero $3.1 \%$ (ver tabla 1).

Tabla 1

Características sociodemográficas de los estudiantes de una universidad privada de Lima Este, 2018.

\begin{tabular}{lll} 
& $\mathrm{N}$ & $\%$ \\
Sexo & & \\
Masculino & 92 & $26.2 \%$ \\
Femenino & 259 & $73.8 \%$ \\
Edad & & \\
16 a 19 años & 108 & $30.8 \%$ \\
20 a 24 años & 204 & $58.1 \%$ \\
25 a más & 39 & $11.1 \%$ \\
Procedencia & & \\
Costa & 204 & $58.1 \%$ \\
Sierra & 108 & $30.8 \%$ \\
Selva & 28 & $8 \%$ \\
Extranjero & 11 & $3.1 \%$ \\
\hline
\end{tabular}


En la tabla 2 se aprecia que el $52.1 \%$ de estudiantes universitarios se encuentra en un nivel moderado, lo que indica que están en riesgo de desarrollar dependencia al celular. Cabe resaltar que el $22.8 \%$ presenta un nivel alto de dependencia al celular, es decir que hacen uso del dispositivo de manera excesiva y cumplen con características principales de dependencia. Por otro lado, existe un $24.8 \%$ que presentan dificultad en controlar el impulso en un nivel alto, es decir les es difícil dejar de usar el celular, mientras que el $57.8 \%$ sufren de abstinencia en un nivel moderado presentando malestar cuando no tiene la posibilidad de utilizar el celular, el $51.3 \%$ tiene la necesidad cada vez mayor de hacer uso del dispositivo (tolerancia) y finalmente, el $53.8 \%$ tiene problemas ocasionados por el uso del celular en un nivel moderado

Tabla 2

Niveles de dependencia al celular en estudiantes universitarios

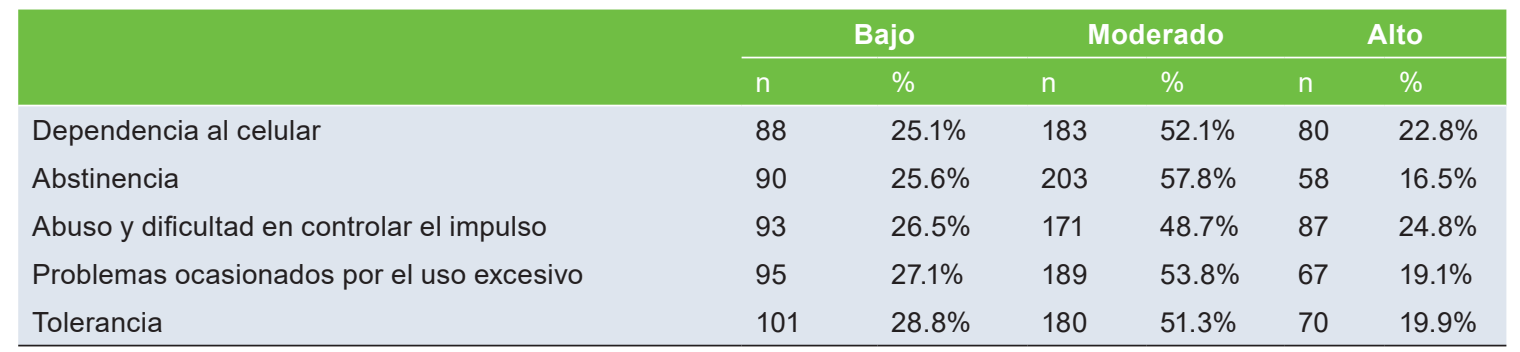

En la tabla 3 se aprecia que el $56.1 \%$ de estudiantes universitarios se encuentra en un nivel bajo, esto indica dificultad para establecer y mantener relaciones interpersonales en diferentes contextos y solo el $14 \%$ tiene la facilidad para relacionarse de manera asertiva.

En cuanto a las dimensiones se aprecia que en el nivel bajo se encuentra el $54.1 \%$ de estudiantes que tienen dificultad en iniciar interacciones positivas, mientras que el $46.2 \%$

Tabla 3

Niveles de habilidades sociales en estudiantes universitarios de los estudiantes les dificulta decir no y hacer peticiones a diversas personas.

Sin embargo, en un nivel alto el $15.1 \%$ de estudiantes tiene facilidad para expresar asertivamente al defender sus derechos como consumidor, de la misma manera facilidad de expresar enfado y desacuerdo con otras personas. Finalmente, a nivel moderado el $47.3 \%$ de los estudiantes tienen tendencia a expresar de forma espontánea y sin ansiedad frente a los demás.

\begin{tabular}{lllllll} 
& \multicolumn{3}{c}{ Bajo } & \multicolumn{2}{c}{ Moderado } & Alto \\
\cline { 2 - 7 } & $n$ & $\%$ & $n$ & $\%$ & $n$ & $\%$ \\
\hline Habilidades sociales & 197 & $56.1 \%$ & 105 & $29.9 \%$ & 49 & $14 \%$ \\
Autoexpresión de situaciones sociales & 133 & $37.9 \%$ & 166 & $47.3 \%$ & 52 & $14.8 \%$ \\
Defensa de los propios derechos como consumidor & 97 & $27.6 \%$ & 201 & $57.3 \%$ & 53 & $15.1 \%$ \\
Expresión de enfado o disconformidad & 106 & $30.2 \%$ & 192 & $54.7 \%$ & 53 & $15.1 \%$ \\
Decir no y cortar interacciones & 162 & $46.2 \%$ & 149 & $42.5 \%$ & 40 & $11.4 \%$ \\
Hacer peticiones & 162 & $46.2 \%$ & 158 & $45 \%$ & 31 & $8.8 \%$ \\
Iniciar interacciones positivas con el sexo opuesto & 190 & $54.1 \%$ & 140 & $39.9 \%$ & 21 & $6 \%$ \\
\hline
\end{tabular}

En la tabla 4, el coeficiente de correlación de Spearman indica que existe relación negativa altamente significativa entre dependencia al celular y habilidades sociales (Rho= -,645, $\mathrm{p}<$ $0.01)$; es decir, un nivel alto de dependencia al celular se asocia a un nivel bajo de habilidades sociales.

Respecto a las dimensiones en abstinencia existe relación negativa y altamente significativa 
$(\mathrm{Rho}=-, 624, p<0.01)$, es decir a niveles altos de abstinencia se asocia un nivel bajo de habilidades sociales. De la misma manera a un nivel alto de abuso y dificultad de controlar el impulso se asocia un nivel bajo de habilidades sociales (Rho= -,526, $\mathrm{p}<0.01$ ). Así mismo un nivel alto de problemas ocasionados por el uso excesivo se asocia un nivel bajo de habilidades sociales $(R h o=-, 595, p<0.01)$. Finalmente, a un nivel alto de tolerancia se asocia a un nivel bajo de habilidades sociales $(\mathrm{Rho}=-, 569, \mathrm{p}<0.01)$.

Tabla 4

Coeficiente de correlación entre dependencia al celular y habilidades sociales

\begin{tabular}{llc} 
Dependencia al celular & \multicolumn{2}{c}{ Habilidades sociales } \\
\cline { 2 - 3 } & Rho & P \\
\hline Dependencia al celular global &,$- 645^{* *}$ &, 000 \\
Abstinencia &,$- 624^{* *}$ &, 000 \\
Abuso y dificultad en controlar el impulso &,$- 526^{* *}$ &, 000 \\
Problemas ocasionados por el uso excesivo &,$- 595^{\star *}$ &, 000 \\
Tolerancia &,$- 569^{* *}$ &, 000
\end{tabular}

** La correlación es significativa al nivel 0.01

\section{DISCUSIÓN}

Esta investigación reporta la existencia de una relación negativa y altamente significativa entre dependencia al celular y habilidades sociales (Rho $\left.=-, 645^{* *} ; p<0.01\right)$. Por lo que se puede interpretar que a mayor dependencia al celular se asocia niveles bajos de habilidades sociales y viceversa. Los resultados coinciden con el estudio en Perú de Chambi y Sucari (2017) quienes encuentran correlación negativa entre dependencia al móvil y habilidades sociales (Rho= - 205, p<0.05) aunque a en esta investigación la relación es débil. Las nuevas tecnologías de información y comunicación (TIC), entre ellas el teléfono celular, se encuentra cada vez más accesible a las familias de cualquier estrato social y su uso es mayor en la población joven, probablemente porque obtiene refuerzo en el contexto social por la aprobación del grupo de iguales, el aumento del reconocimiento y la integración en el grupo (Arias Gallegos y Rodríguez, 2012). Si bien es cierto, estos dispositivos han traído múltiples beneficios no se puede negar que también han originado hábitos de comportamiento encaminados al abuso y a cierta dependencia (Sánchez, Beranuy, Castellana, Chamarro y Oberst, 2008). Las consecuencias presentadas en el uso excesivo del celular llevan a alejarse de sus amistades, reduciendo las interacciones, haciéndose menos sociable y poniendo en riesgo sus habilidades sociales (Tejeiro, Pelegrina y Gómez, 2009) como se refiere en una investigación: Se encontró déficit de habilidades sociales entre aquellos que presentan un uso problemático (García et al., 2008). De acuerdo a Echeburúa y Corral, (1994) refieren que la condición adictiva provoca problemas en la vida cotidiana de las personas afectadas y en sus relaciones a nivel familiar, laboral y social. Por otro lado, Putnam (2001) indica que aquellas personas que poseen recursos sociales, tienen más oportunidades para interacciones cara a cara y menos probabilidad de recurrir a los celulares como escape de la interacción directa.

Es importante mencionar que la población joven es identificada como la que presenta mayores niveles de uso de las nuevas tecnologías y es además la más vulnerable a la aparición de diversas conductas adictivas (Muñoz, Rivas y Agustin, 2005). Específicamente con respecto al celular Sánchez-Carbonell y Beranuy (2007) refieren que lo atractivo de este dispositivo son: sentirse miembro de un grupo, la construcción de identidades, los juegos sexuales y de galanteo, el bienestar psicológico, la inmediatez, la accesibilidad y la comunicación mediante la escritura, las cuales tienen las propiedades reforzadoras que dan las sustancias adictivas. Esto conlleva a crear nuevos estilos que dificultan las relaciones no solo con personas con las que se tiene un primer contacto sino también con aquellas con las que más se frecuenta, llegando a priorizar la comunicación a través del celular y alterando las relaciones sociales (Bianchi y Phillips, 2005). 
Con relación al primer objetivo específico, se evidencia que existe relación negativa y altamente significativa entre abstinencia y habilidades sociales (Rho=-,624**, $p<0.01$ ), de tal manera que los niveles altos de abstinencia se asocian con la dificultad en relacionarse de manera hábil en las interacciones sociales y viceversa. La teoría del aprendizaje, sustenta que las conductas adictivas se activan en un inicio mediante reforzador positivo (experiencia de placer o euforia) pero cuando la conducta se mantiene, el reforzador termina siendo negativo, con el objetivo de no experimentar el malestar, evitando el síndrome de abstinencia (alivio de la tensión emocional) producto de la falta de uso del dispositivo (Echeburúa, 1999). En la abstinencia se dan reacciones fisiológicas o emocionales como: alteraciones en el humor, irritabilidad, impaciencia, inquietud o angustia (Gámez, Muñoz y Fernández, 2009), por lo que se puede deducir que estas características pueden limitar la instalación o el progreso de las habilidades sociales las que son susceptibles de aprendizaje y desarrollo (Bisquerra, 2003). Además, Gismero en la conceptualización de habilidades sociales refiere que el individuo expresa en un contexto interpersonal sus necesidades, sentimientos, preferencias, opiniones o derechos sin ansiedad excesiva y de manera asertiva, respetando todo ello en los demás, que trae como consecuencia el auto reforzamiento y maximiza la probabilidad de conseguir refuerzo externo. Mientras que en la dependencia pasa lo contrario no hay expresión en un contexto interpersonal y si los hay son expresados con ansiedad excesiva y no de manera asertiva por la presencia de irritabilidad consecuencia de la abstinencia, y por último la consecuencia no es un reforzamiento positivo externo (interpersonal) sino un reforzamiento negativo para huir del malestar.

En el segundo objetivo específico se evidencia relación negativa moderada y altamente significativa entre abuso y dificultad de controlar el impulso con habilidades sociales (Rho $=-.526^{* *}, p<0.01$ ) por lo tanto, cuando los estudiantes universitarios no logran dejar de utilizar el celular (falta de control conductual) y hacen uso excesivo del dispositivo en cualquier lugar o situación, tendrán menos probabilidades de desarrollar las habilidades sociales como: dificultad en iniciar interacciones positivas con el sexo opuesto o dificultad en hacer peticiones tanto a amistades como a desconocidos. Lo manifestado en líneas anteriores, se comprueba con lo dicho por el Ministerio de salud (2005) "Los seres humanos progresivamente aprenden competencias en las interacciones sociales cotidianas" es decir que las habilidades sociales son aprendidas en el intercambio entre uno con los demás y entre los demás para con uno.

Así mismo, en el tercer objetivo se evidencia relación negativa y altamente significativa entre problemas ocasionados por el uso excesivo y habilidades sociales (Rho=-,624** $p<0.01$ ), significa que los estudiantes que tienen mayores consecuencias negativas por el uso excesivo del celular tanto a nivel personal , familiar y social, tendrán más dificultades para desarrollar sus habilidades sociales ya que en la dependencia la realización de las conductas se vuelve automática, apoderándose en ellos las emociones e impulsos, además desaparece el control cognitivo y autocritico, solo está presente la búsqueda de la recompensa inmediata a través de la realización de la conducta sobre el acierto o error de la decisión (Echeburrúa y Paz de Corral 2010). Por lo tanto, la conducta es regulada por la frecuencia de consumo, intensidad, tiempo empleado en la misma y dinero invertido en su realización (Cía, 2013) lo que conlleva a presentar problemas como interferencia con actividades importantes y problemas en las relaciones sociales y familiares. Se puede inferir por lo ya expuesto que este actuar constante puede consecuentemente llevar a no desarrollar la capacidad de expresar emociones de manera apropiada a través del lenguaje y la comunicación verbal (Rodríguez et al. 2012). Así mismo, no se cumpliría lo dicho por Caballo (1993) al conceptualizar las habilidades sociales como la capacidad de saber manejar las emociones, tener comunicación abierta, aceptada por los demás, es saber cuándo actuar, cuando hablar, esto basado en una manifestación conductual cognitivo y emocional.

Finalmente, en el cuarto objetivo se evidencia relación negativa y altamente significativa entre tolerancia y habilidades sociales (Rho=-,569**, $p<0.01$ ) a tenor de lo encontrado, el estudiante universitario con dependencia presenta más necesidad de incrementar el uso del dispositivo para satisfacerse o lograr el efecto agradable (euforia) esto sugiere la asociación con menores niveles de habilidades sociales.

La explicación encontrada en la literatura refiere que las personas con adicción a las nuevas tecnologías al mantener la conducta adictiva 
tienen un sentimiento placentero parecido a cualquier adicción (Labrador y Villadangos, 2010). Por otro lado, el incremento del uso del celular trae como consecuencia el aislamiento social y esta falta de interacción dificulta el desarrollo de habilidades sociales ya que estas son aprendidas lo que implica que todo lo que es aprendido es susceptible de ser modificado (Ortego, López y Álvarez, 2011).

Declaración de financiamiento y de conflictos de interés:

El estudio fue financiado por los autores, quienes declaran no tener conflictos de interés.

\section{Correspondencia}

Diana De la Cruz Sandoval

Correo electrónico:

delacruzsandovaldianamaribel@gmail.com

Luz Torres Zárate

Correo electrónico: luzlilitorres@hotmail.com

Elí Yánac Cierto

Correo electrónico: eli.yanac@upeu.edu.pe

\section{REFERENCIAS BIBLIOGRÁFICAS}

Agencia Peruana de Noticias. (2013). Adicción al teléfono móvil afecta al $40 \%$ de usuarios en Perú. Andina. Recuperado de: https://andina. pe/agencia/noticia-adiccion-al-telefono-movilafecta-al-40-usuarios-peru-446589.aspx

Arias, C. M. Ávila, L. F. Enciso, J. C. Garzón, S. J. matías, L. M. Peralta, D. A. Preciado, L. C. Torres, D. V., Torres, Á., Felipe, L., Abril, E., Cecilia, J., Cruz, G. \& Lozano, J. T. (2011). «Influencia De Los Smartphones En Los Estilos De Vida De Los Jovenes Universitarios Y Jovenes Ejecutivos En La Ciudad De Bogota». (Tesis de pregrado). Universidad Jorge Tadeo Lozano, Bogota. Recuperado de: https://www.utadeo.edu.co/files/ collections/documents/field_attached_file/ influencia_de_los_smartphones_en_los_estilos_ de_vida_de_los_jovenes_universitarios_y_ jovenes_ejecut ivos_en_la_ciudad_de_b1.pdf.

Bianchi, A. y Phillips, J. (2005). Psychological predictors of problem mobile phone use. Cyberpsychology \& Behavior, 8, 39-51. Recuperado de: https:// www.semanticscholar.org/paper/PsychologicalPredictors-of-Problem-Mobile-Phone-Bianchi-Ph illips/683f1948bc6ccbe7713a6f723178c6ea2c $5 \mathrm{~d} 2046$
Bisquerra, R. (2003). "Educación emocional y competencias básicas para la vida". Revista de Investigación Ed u c a t i $v$ a,

21 (1) 7-43. Recuperado de: https:// www.researchgate.net/publication/41570276_ Educacion_emocional_y_competenci as_basicas_ para_la_vida

Caballo, E. (1993). Manual de evaluación y entrenamiento de las Habilidades Sociales. Recuperado de: https://www.navarra.es/ NR/rdonlyres/BBF448C9-11C6-48B3-A574558618E4E4F2/138615/Cap_04_97122.pdf

Carbonell, X., Fúster, H., Chamarro, A., \& Oberst, U. (2012). Adicción a Internet y Móvil: Una Revisión De Estudios Empíricos Españoles. Papeles del Psicólogo, 33 (2), 82-89. Recuperado de: http:// www.papelesdelpsicologo.es/pdf/2096.pdf

Chambi, S., \& Sucari, B. (2017). Adicción a internet, dependencia al móvil, impulsividad y habilidades sociales en pre-universitarios de la Institución Educativa Privada Claudio Galeno, Juliaca. Tesis de licenciatura. Recuperado de: http://repositorio. upeu.edu.pe/bitstream/UPEU/105/1/Bach. Evelyn M. Cotrado Macedo y Ubaldina J. Infantes Mamani.pdf

Chóliz, M. (2012). Mobile-phone addiction in adolescence: The Test of Mobile Phone Dependence (TMD). Progress in Health Sciences, 2(1). Recuperdo de: https://www.researchgate. net/publication/284690452_Mobilephone addiction_in_adolesc ence_The_Test_of_Mobile_Phone_Dependence_TMD

Cia, A. (2013). Las adicciones no relacionadas a sustancias (DSM-5, APA, 2013): un primer paso hacia la inclusión de las Adicciones Conductuales en las clasificaciones categoriales vigentes. Revista médica herediana Recuperado de: http:// www.upch.edu.pe/vrinve/dugic/revistas/index. php/RNP/article/view/1169/1201

Echeburúa, E. \& Corral, P. (2009). Las adicciones con o sin droga: una patología de la libertad. Madrid: $\quad$ Pirámide.. Revista versión online. Recuperado de: https://dialnet.unirioja.es/servlet/ articulo?codigo $=5027197$.

García, A., Terol, C., Nieto, M., Lledó, A., Sánchez, S., Aragón, M; \& Sitges, E. (2008). Uso y abuso de Internet en jóvenes universitarios. Adicciones, 20, 131-142. Revista adicciones. Recuperado de: http://www.redalyc.org/pdf/2891/289122057005. pdf

Labrador, F.y Villadangos, S. (2010). Menores ynuevas tecnologías: conductas indicadoras de posible problema de adicción. Revista Psicothema, 22(2), 180-188. Recuperado de: https://www.redalyc. org/articulo.oa?id=72712496002 
Mejía, G., Paz, J., \& Quinteros, D. (2014). Adicción a facebook y habilidades sociales en estudiantes de una universidad privada de Lima. Revista Cientifica de La Salud, 7-15. Recuperado de: http://revistascientificas.upeu.edu.pe/index.php/ rc_salud/article/view/209

Mejía, G., Paz, J., \& Quinteros, D. (2014). Adicción a facebook y habilidades sociales en estudiantes de una universidad privada de Lima. Revista Cientifica de La Salud, 7-15. Recuperado de: http://revistascientificas.upeu.edu.pe/index.php/ rc_salud/article/view/209

Ministerio de salud (2005). Manual de habilidades sociales en adolescentes escolares .Dirección General de Promoción de la Salud. Dirección Ejecutiva de Educación para la Salud; Instituto Especializado de Salud Mental "Honorio Delgado/ Hideyo Noguchi". Recuperado de: http://bvs. minsa.gob.pe/local/PROMOCION/170_adolec. pdf

Morales, L. (2012). Adicción al teléfono móvil o celular. Rev. Psicólogos, 2(1), 94-102. Recuperado de: https://docplayer.es/3321662-Adiccion-altelefono-movil-o-celular.html

Muñoz, M; Rivas, J; \& Agustín, S. (2005). La adicción al teléfono móvil. Psicología Conductual, 13, 481493. Recuperado de: https://dialnet.unirioja.es/ servlet/articulo?codigo $=1404332$

Niño, J. (2013). La Importancia del Teléfono Móvil Para La Comunicación Publicitaria. Historia y comunicación. ISSN Historia y comunicación social, $\quad$ 18. 581-590.R e cuperado de: derevistas.ucm.es/index.php/hics/article/ download/43990/41596

Ortego, M; López; S \& Álvarez, M. (2011). Ciencias Psicosociales I. Open course ware Recuperado de: $\quad$ https://ocw.unican.es/course/view. php?id=182\&section $=1$

Putnam, R. (2001). La comunidad próspera. El capital social y la vida pública" Zona Abierta: Madrid. Dialnet Recuperado de: https:// flacsoandes.edu.ec/sites/default/files/agora/ files/1252374169.hintzecapital_social__y_ estrategias_de_supervivencia._reflexiones_ sobre_el_capitall_0.pdf

Ruiz, J., Sánchez, J., \& Trujillo, J. (2016). Utilización de Internet y dependencia a teléfonos móviles en adolescentes TT - Using Internet and dependence on mobile phones in. Recuperado de: http://www.scielo.org.co/pdf/rlcs/v14n2/ v14n2a33.pdf

Salvador, D. (2017). Adicción a internet y habilidades sociales en estudiantes de secundaria de la institución educativa Internacional (Tesis de Maestría. Universidad Cesar Vallejo). Recuperado de http://repositorio.ucv.edu.pe/bitstream/handle/ UCV/14122/Romo_SDS.pdf? sequence $=1$ \& isAllowed=y

Sánchez, X; Beranuy, M; Castellana, M; Chamorro, A; \& Oberst, U. (2008). La adicción a intenet y al móvil: ¿moda o trastorno?. Revista de adicciones, 20(2), 149-159. Recuperado de: https://www. redalyc.org/html/2891/289122057007/

Tejeiro, R; Pelegrina, M; \& Gómez, J. (2009). Efectos Psicosociales de los Videojuegos, Comunicación, Revista de Comunicación, 1(7), 235-250. Recuperado de http://www.revistacomunicacion. org/pdf/n7/articulos/a16_Efectos_psi cosociales_ de_los_videojuegos.

Telesur, (2017). Adicción al celular es parecida a dependencia a las drogas. Telesur Recuperado de: https://www.telesurtv.net/news/Adiccional-celular-es-parecida-a-dependencia-a-lasdrogas-20171111-0036.html

Viracocha, C. (2017). Uso de dispositivos móviles $y$ desarrollo de habilidades sociales en adolescente. (Tesis de licenciatura. Universidad central del ecuador) Recuperado de: http://www. dspace.uce.edu.ec/bitstream/25000/12753/1/TUCE-0007-PC009-2017.pdf

Recibido: 15 de marzo Aceptado: 20 de mayo 